\title{
Energía Calorífica Necesaria, Durante la Soldadura en Servicio de Tuberías para el Transporte de Petróleo
}

\author{
(Necessary Calorific Energy, During the In-Service Welding of Pipelines for Petroleum Transport)
}

Félix Ramos Morales ${ }^{1}$, Alejandro Duffus Scott ${ }^{1}$, Manuel Rodríguez Pérez¹, Eduardo M. Díaz Cedré1, Juan A. Pozo Morejón ${ }^{1}$. ${ }^{1}$ Centro de Investigaciones de Soldadura. Universidad Central "Marta Abreu" de Las Villas. Santa Clara, Villa Clara, Cuba, felixrm@uclv.edu.cu

\section{Resumen}

En el presente artículo, empleando el análisis por elementos finitos, se estudió el comportamiento térmico durante la soldadura de reparación, en condiciones de servicio, de un tubo que transporta petróleo. Se obtuvieron ecuaciones de regresión que relacionan a la temperatura máxima en la superficie interna del tubo y al tiempo de enfriamiento desde $800{ }^{\circ} \mathrm{C}$ hasta $500{ }^{\circ} \mathrm{C}$ de un punto situado en la zona afectada por el calor (ZAC), parámetros que gobiernan la ocurrencia de perforaciones en la pared del tubo y agrietamiento en frí respectivamente, con el calor de entrada de la soldadura, la temperatura de precalentamiento y el coeficiente de transferencia de calor por convección, variables que definen el comportamiento térmico durante la ejecución del proceso de soldadura. Se probó la existencia de una zona en que se satisfacen simultáneamente las ecuaciones obtenidas, para diferentes combinaciones de las variables relacionadas. Se presentaron nomogramas con un importante valor práctico, desarrollados a partir de la solución de las ecuaciones obtenidas.

Palabras claves: reparación, refuerzos, perforación, agrietamiento en frio, elementos finitos.

\begin{abstract}
The thermal behavior during in service repair welding of oil transportation pipes was studied by finite element analysis in the present paper. Regression equations that relate peak temperature at the inner surface of the pipe and cooling time between 800 and $500^{\circ} \mathrm{C}$ in the heat affected zone to the welding heat input, preheat temperature, and convection heat transfer coefficient were obtained. The former parameters govern, respectively, the possibility of burn through and cold cracking, and the latter parameters define the thermal behavior during welding. The existence of conditions that simultaneously satisfy the obtained equations, for different combinations of related variables, was proved. Graphical representations of relevant practical importance that were developed from the solution of obtained equations are presented.
\end{abstract}

Keywords: reparation, sleeves, burn through, cold cracking, finite elements.

\section{Introducción}

Apesarde la implementación de estrictas regulaciones acerca de: la construcción, explotación, inspección, mantenimiento y reparación de las tuberías que transportan petróleo, estas fallan causando pérdidas humanas y económicas [1]. Las causas de estas fallas son variadas y pueden estar relacionadas a la actividad humana o inducidas por largos períodos de servicio. Específicamente sobre las fallas inducidas por el servicio, las causas principales son: pérdida de espesor debido a la corrosión y agrietamiento originado por fragilidad por hidrógeno, corrosión bajo tensión o fatiga. Estudios desarrollados por Kiefner [2] muestran que entre 1986 y 1999, en los Estados Unidos, el 12 $\%$ de las fallas en tuberías estuvieron relacionadas a defectos de soldadura.

Frente a la presencia de daños en tuberías, que pueden conducir a una falla catastrófica, se hace necesario reparar y esta decisión tiene una fuerte justificación económica. Estudios de

(Recebido em 29/01/2008; Texto Final em 07/01/2009).
Palmer - Jones [3] revelan que la reparación de un tubo, con un diámetro entre $406,4 \mathrm{~mm}$ y $610 \mathrm{~mm}$, puede ahorrar entre el 10 $\%$ y el $50 \%$ del costo de una tubería nueva.

Hay varias alternativas para realizar la reparación de una tubería y cada una tiene aspectos favorables y desfavorables, por ejemplo:

1. Pequeños parches metálicos soldados a la superficie exterior del tubo: De acuerdo a investigaciones previas desarrolladas por Fazzini [4], este método de reparación es ampliamente empleado en la industria del petróleo. La principal limitación que posee es que, el largo del parche no puede exceder la mitad del diámetro del tubo [5] y entonces defectos extensos no pueden ser reparados por este método.

2. Refuerzos metálicos circunferenciales, atornillados alrededor de la superficie exterior del tubo [6]: Con este método se pueden evitar los defectos asociados al proceso de soldadura pero no se puede detener la propagación de grietas longitudinales a través de la pared del tubo.

3. Refuerzos de composite (llamados también "clock spring"): Con estos refuerzos el tubo es protegido de la corrosión 
externa y el desgaste, pero no se recomienda el uso de estos refuerzos para reparar defectos agudos, como grietas [7].

4. Refuerzos metálicos circunferenciales, soldados alrededor de la superficie externa del tubo: Este método es catalogado, por Bruce [8], como la opción de reparación más común. Los refuerzos evitan la propagación de grietas longitudinales y son diseñados para soportar, al menos, la presión de diseño de la tubería. Durante la reparación de una tubería en condiciones de servicio, por este método, es necesario considerar la posible ocurrencia de dos fenómenos [9]: perforaciones y agrietamiento en frío.

Ambos fenómenos (las perforaciones de la pared del tubo y el agrietamiento en frío) son igualmente indeseables y opuestos, esto significa que las acciones que evitan la ocurrencia de uno de ellos, favorecen la ocurrencia del otro. En ambos tienen una pronunciada influencia las variables relacionadas a la energía calorífica que penetra a la pieza y las condiciones de disipación del calor (calor de entrada de la soldadura, temperatura de precalentamiento y el coeficiente de transferencia de calor por convección).

El método de los elementos finitos ha sido ampliamente empleado para predecir la posibilidad de ocurrencia de perforaciones y agrietamiento en frío, durante la soldadura en servicio de tuberías. Hay en la literatura especializada muchos artículos sobre este tema, por ejemplo:

1. Cisilino [10] obtiene los campos de temperatura en un tubo de acero X 52, el cual transportaba gas natural y fue soldado por proceso de soldadura manual con electrodo revestido (SMAW). El modelo de elementos finitos empleado es tridimensional (3D). El criterio límite establecido para seleccionar el régimen adecuado fue: la temperatura en la superficie interna del tubo no puede exceder $982{ }^{\circ} \mathrm{C}$. En este artículo no fue considerada la posibilidad de ocurrencia de agrietamiento en frío y este aspecto representa una limitación de la solución obtenida.

2. Bang [11] desarrolló un modelo axisimétrico, bidimensional (2D), de elementos finitos para simular la soldadura de un refuerzo metálico circunferencial a un tubo de acero X 65 . En los modelos desarrollados fueron considerados dos criterios límites: $982{ }^{\circ} \mathrm{C}$ como temperatura máxima en la superficie interna del tubo y $350 \mathrm{HV}$ como la dureza máxima en la zona afectada por el calor (ZAC) para hidrocarburos no amargos y $250 \mathrm{HV}$ como dureza límite para hidrocarburos amargos. En este artículo fue considerada, también, la termodependencia de las propiedades físicas y mecánicas del acero.

3. Oddy [12] reconoce como una necesidad operacional la necesidad de soldar bifurcaciones en las tuberías de gas, con estas totalmente presurizadas. También en este artículo se mencionó la necesidad de establecer un calor de entrada de la soldadura lo suficientemente bajo para evitar perforaciones en el tubo pero no tanto que pueda ocurrir agrietamiento en frío. Fue mencionada la dificultad de encontrar datas que muestren las propiedades de los aceros de tuberías en función de la temperatura.

Durante los últimos años han sido desarrollados algunos software con propósitos comerciales y/o académicos. En Internet puede ser encontrado el "Master Code" de Yurioka
[13]. Este código permite calcular el tiempo de enfriamiento desde $800{ }^{\circ} \mathrm{C}$ hasta $500{ }^{\circ} \mathrm{C}$ (conocido como t $8 / 5$ ), la máxima dureza en la zona afectada por el calor (ZAC), la temperatura de precalentamiento mínima necesaria, entre otros parámetros. La limitación de este código para aplicarlo al caso de la soldadura en servicio de tuberías es que no es posible considerar el alto valor del coeficiente de transferencia de calor por convección. Las expresiones del Master Code de Yurioka fueron deducidas para condiciones de soldadura donde la conducción es el principal mecanismo de disipación del calor.

Como resultado de la colaboración entre el "Cooperative Research Centre for Welded Structures" de Australia y la "Australian Pipeline Industry", fue desarrollado un nuevo software para soldadura en servicio. Este software estima condiciones seguras de soldadura, para determinadas condiciones en la tubería, mediante:

1. Estableciendo una indicación de los límites superior e inferior del calor de entrada de la soldadura.

2. Determinando la dureza en la ZAC que resultará de un calor de entrada dado.

3. Determinando el calor de entrada que va a crear un riesgo de perforaciones.

El antes mencionado software es el resultado de una base de datos creada a partir de la solución de un modelo, 3D, de elementos finitos.

Basado en las investigaciones previas, el presente artículo pretende evaluar la influencia del calor de entrada de la soldadura, la temperatura de precalentamiento y el coeficiente de transferencia de calor por convección sobre la ocurrencia de perforaciones y agrietamiento en frío, con vistas a establecer ecuaciones que expresen un compromiso para que estos fenómenos indeseables no se manifiesten, para aceros de tuberías con diferente composición química. Las características geométricas de la tubería considerada son $6.4 \mathrm{~mm}$ de espesor de la pared del tubo y $610 \mathrm{~mm}$ de diámetro.

\section{Materiales y métodos}

\subsection{El método de los elementos finitos para simular, la soldadura en condiciones de servicio de un tubo}

El tubo elegido para el estudio tiene un diámetro exterior de $610 \mathrm{~mm}$ y espesor de $6,4 \mathrm{~mm}$. La selección de estas dimensiones se realiza teniendo en cuenta que, de acuerdo a la norma API 1104 [9], para este espesor y menores, existe gran riesgo de ocurrencia de perforación de la pared del tubo cuando la reparación por soldadura se realiza en condiciones de servicio; en cuanto al diámetro, este se encuentra dentro del rango de los más empleados para el transporte de petróleo a nivel mundial.

Para simular la reparación por soldadura de la tubería, en condiciones de servicio, fue creado un modelo tridimensional (3D) de elementos finitos, que representa una sección reforzada del tubo. El software empleado para la solución del modelo fue el Cosmos DesignSTAR v. 4.0.

Por cuestiones inherentes al tiempo computacional (cuyo aumento implica una elevación del costo de la investigación) y al número límite de elementos y nodos que admiten los programas 
de elementos finitos, al crear el modelo solo se extrae una sección del tubo y se desprecia su curvatura, tal y como muestra la Figura 1. Estas consideraciones no sacrifican la calidad de los resultados que se obtienen.

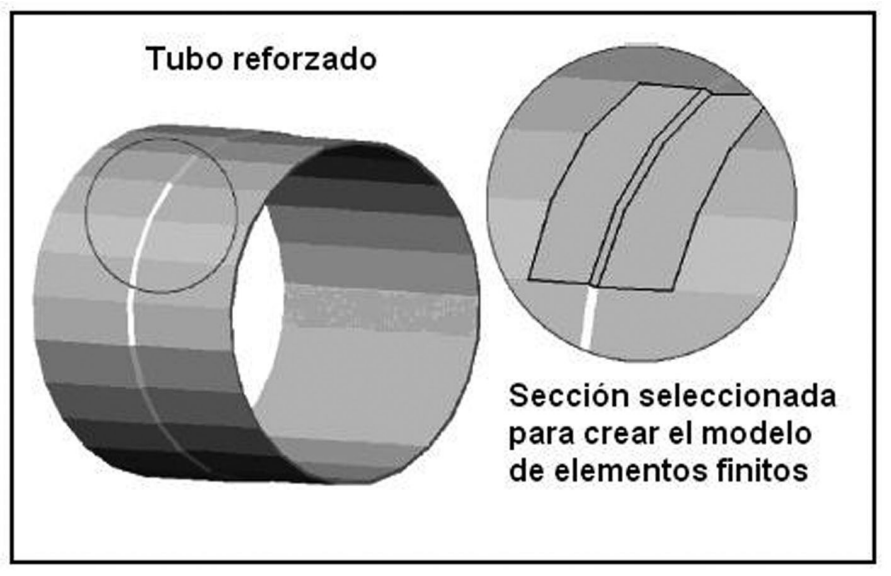

Figura 1. Tubo reforzado y sección seleccionada para la creación del modelo de elementos finitos.

Ignorar la curvatura se justifica a partir de artículos previamente publicados [10] y de acuerdo a Pavlov [14] que plantea que la ecuación para la transmisión del calor, deducida para una placa plana, puede ser empleada para calcular la transmisión del calor a través de una placa cilíndrica, si la Ecuación 1 es válida:

$$
d_{\text {int }}>0,5 \times d_{\text {ext }}
$$

Donde: $\mathrm{d}_{\text {int }} \mathrm{y} \mathrm{d}_{\text {ext }}$ representan los diámetros interior y exterior de la tubería, respectivamente.

En el modelo fue ignorada la holgura tubo - refuerzo, porque esta no va a tener una significativa influencia en la distribución de temperaturas. El mallado del modelo fue hecho empleando elementos finitos de orden superior (sólido, tetraédrico, parabólico con 10 nodos). Se realizó un análisis de convergencia para 4 condiciones diferentes de mallado, con vistas a eliminar cualquier influencia significativa de la dimensión de la malla sobre el resultado obtenido. Un grupo de suposiciones consideradas en el modelo de elementos finitos, fueron validadas previamente por los autores [15].

Las variables consideradas fueron: calor de entrada de la soldadura, temperatura de precalentamiento y coeficiente para la transferencia de calor por convección; porque estas variables definen el comportamiento térmico durante la soldadura [16]. Los valores de las variables fueron establecidos entre límites mínimos y máximos, de acuerdo con recomendaciones operativas y tecnológicas de la industria del petróleo.

Con vistas a comprobar la validez de las suposiciones asumidas en el modelo, se realizó una corrida con datos similares a los empleados por Cisilino [10] y los resultados obtenidos mostraron un alto nivel de correspondencia, lo que se muestra en la Tabla 1.

Tabla 1. Correspondencia entre los resultados del modelo de Cisilino y la presente investigación.

\begin{tabular}{|c|c|c|c|}
\hline \multicolumn{5}{|c|}{$\mathbf{6 , 4} \mathbf{~ m m}$ de espesor del tubo } \\
\hline Profundidad $(\mathbf{m m})$ & $\begin{array}{c}\text { Temperatura }\left({ }^{\circ} \mathbf{C}\right) \\
\text { según Cisilino et. al }\end{array}$ & $\begin{array}{c}\text { Temperatura }\left({ }^{\circ} \mathbf{C}\right) \\
\text { según investigación }\end{array}$ & $\begin{array}{c}\text { \% Error respecto a } \\
\text { Cisilino et. al }\end{array}$ \\
\hline 2,35 & 1430 & 1365 & $4,55 \%$ (por defecto) \\
\hline 2,70 & 1260 & 1227 & $2,62 \%$ (por defecto) \\
\hline 3,45 & 982 & 989 & $0,71 \%$ (por exceso) \\
\hline
\end{tabular}

\section{1.1. Establecimiento del calor de entrada de la soldadura} (CE)

El proceso seleccionado para desarrollar la reparación es el de soldadura manual por arco eléctrico con electrodo revestido (SMAW). El cálculo del calor de entrada (CE) se realiza partiendo de los límites en las características operativas, mostradas en la Tabla 2, del electrodo E 7018 de $2,4 \mathrm{~mm}$ de diámetro $[17,18]$ y considerando una eficiencia del proceso de 0,7 [18]. La velocidad de soldadura teórica fue determinada con vistas a depositar el metal de la costura en una sola pasada.

Tabla 2. Características operativas límites del electrodo E 7018 de 2,4 mm de diámetro.

\begin{tabular}{|c|c|c|}
\hline Parámetro & Valor inferior & Valor superior \\
\hline Corriente de Soldadura $(\mathrm{A})$ & 70 & 110 \\
\hline Voltaje de arco $(\mathrm{V})$ & 18 & 24 \\
\hline Razón de Deposición $\left(\mathrm{kg} \cdot \mathrm{h}^{-1}\right)$ & 0,623 & 0,786 \\
\hline Velocidad de soldadura $\left(\mathrm{mm}^{-1} \mathrm{~s}^{-1}\right)$ & 1,09 & 1,38 \\
\hline
\end{tabular}


Los valores de calor de entrada considerados en el presente artículo fueron de $810 \mathrm{~J} . \mathrm{mm}^{-1}$ (valor inferior), $1217 \mathrm{~J} . \mathrm{mm}^{-1}$ (valor intermedio) y $1625 \mathrm{~J} . \mathrm{mm}^{-1}$ (valor superior); la Ecuación 2 fue la empleada en el cálculo del calor de entrada [18].

$$
C E=\frac{\eta \times U \times I}{V}
$$

Donde: $\mathrm{CE}$ - calor de entrada de la soldadura; $\eta$ - eficiencia del proceso de soldadura: U - voltaje de arco; I - corriente de soldadura; V - velocidad de soldadura.

La longitud de cordón considerada fue calculada a partir de la eficiencia de deposición y la masa del electrodo, empleando la Ecuación 3 [18]. La longitud resultante fue de $55 \mathrm{~mm}$ de metal depositado continuamente.

$$
E D=\frac{m_{\text {metal depositado }}}{m_{\text {electrodo }}} \times 100 \%
$$

Donde: ED - eficiencia de deposición; $m_{\text {metal-depositado }}$ - masa de metal depositado; melectrodo - masa del electrodo empleado.

\section{1.2. Establecimiento de la temperatura de precalentamiento (TP)}

El límite inferior de la temperatura de precalentamiento (TP), que fue considerado en la presente investigación coincide con la mínima temperatura a la que fluye el fluido a través de la tubería, la cual va a estar cercana a $50{ }^{\circ} \mathrm{C}$ en muchos sistemas de tuberías. Esta consideración fue hecha porque la conducción del calor a través del tubo va a equilibrar la temperatura de este con la del fluido. Para la determinación del límite superior de la temperatura de precalentamiento, primero, fueron analizados, los métodos tradicionales como: el método de control de hidrógeno y el método de control de la dureza en la zona afectada por el calor [19]. Ambos métodos resultan en que no es necesario precalentar de acuerdo a la composición química del acero, el espesor del tubo y otros factores, pero estos métodos no consideran la existencia de un intenso enfriamiento debido al flujo del fluido en la tubería y que la reparación se realiza en tuberías viejas, generalmente; entonces estos resultados no pueden ser considerados aceptables.

Considerando que precalentar a altas temperaturas reduce el nivel máximo de hidrógeno acumulado y debido al importante rol del hidrógeno en el agrietamiento en frío [20], fue necesario investigar por recomendaciones en la literatura especializada. Los principales resultados de esta investigación fueron:

1. En las tuberías con una larga vida en servicio la intensidad de atrapamiento de hidrógeno puede alcanzar siete veces la capacidad de atrapamiento de una tubería nueva [21]; entonces el contenido de hidrógeno residual y difusible en un tubo viejo puede se muy alto y la tendencia al agrietamiento en frío se incrementa.

2. Con vistas a evitar el agrietamiento en frío en aceros clasificados como X60 y X65 (con un número Pcm de aproximadamente 0.20 ) es necesario un tiempo de enfriamiento entre 800 y 500 ${ }^{\circ} \mathrm{C}$ menor que 10 segundos [22].
3. De acuerdo a Yurioka [23] se estima que para una temperatura de precalentamiento de $200{ }^{\circ} \mathrm{C}$ la relación entre hidrógeno local / hidrógeno inicial en el metal de la costura puede ser mantenida por debajo de 0,5 durante el intervalo de enfriamiento, precisamente desde la terminación de la soldadura hasta más de 14 horas después de culminada. Esto reduce la posibilidad de ocurrencia de agrietamiento en frío.

Por las razones previamente mencionadas, en la presente investigación, fue considerado $200{ }^{\circ} \mathrm{C}$ como valor mayor de la temperatura de precalentamiento.

Expertos consideran que en la práctica común es muy difícil alcanzar y mantener temperaturas de precalentamiento mayores que $150{ }^{\circ} \mathrm{C}$, debido a la rápida transferencia del calor hacia el contenido del tubo, particularmente para secciones de espesor fino. Sin embargo, en la presente investigación se mantuvo $200{ }^{\circ} \mathrm{C}$ como el límite superior de la temperatura de precalentamiento porque esto expande el rango de validez de las ecuaciones obtenidas, los resultados son perfectamente válidos para una temperatura de precalentamiento de $150{ }^{\circ} \mathrm{C} \mathrm{y}$, como es presentado posteriormente, muchos aceros solo satisfacen las que ecuaciones que fueron obtenidas si la temperatura de precalentamiento es de $200{ }^{\circ} \mathrm{C}$. El valor medio, del rango de temperaturas de precalentamiento consideradas es de $125^{\circ} \mathrm{C}$.

\section{1. 3. Establecimiento del coeficiente de transferencia de calor por convección (CP)}

La Ecuación 4 es la ecuación general que describe la transferencia de calor, por convección, en un tubo con un fluido fluyendo internamente.

$$
N=C \times R^{m} \times P^{n}
$$

Donde: N, R y P son los grupos adimensionales de Nusselt, Reynolds y Prandtl respectivamente; C, m y n son constantes experimentales.

Para la determinación de los valores límites del coeficiente de transferencia de calor por convección (CP) en la superficie interior del tubo, donde un líquido viscoso (petróleo) está fluyendo, la Ecuación que es empleada es denotada como 5 en este artículo [24]. Las propiedades del fluido, mostradas en la Tabla 3, fueron calculadas considerando la temperatura del fluido (entre $50{ }^{\circ} \mathrm{C}$ y $90{ }^{\circ} \mathrm{C}$ ). La velocidad de circulación del fluido se consideró entre $1 \mathrm{~m} \cdot \mathrm{s}^{-1}$ y $3 \mathrm{~m} \cdot \mathrm{s}^{-1}$.

$$
\frac{C P D}{k f}=0,027 \times\left(\frac{D v \rho}{\mu}\right)^{0,8} \times\left(\frac{C_{P} \mu}{k f}\right)^{1 / 3} \times\left(\frac{\mu}{\mu_{P}}\right)^{0,14}
$$

Donde: $\mathrm{CP}$ - coeficiente de transferencia de calor por convección; $\mathrm{D}$ - diámetro del tubo; $\mathrm{kf}$-conductividad térmica del fluido; $v$-velocidad del fluido; $\rho$-densidad del fluido; $\mathrm{C}_{\mathrm{P}}-$ calor específico del fluido a presión constante; $\mu$-viscosidad del fluido a la temperatura del fluido; $\mu_{\mathrm{p}}$ - viscosidad del fluido a la temperatura de la superficie del tubo. 
Tabla 3. Propiedades físicas del fluido en función de la temperatura de circulación.

\begin{tabular}{|c|c|c|}
\hline Propiedad & $\begin{array}{c}\text { Valor } \\
\text { inferior }\end{array}$ & $\begin{array}{c}\text { Valor } \\
\text { superior }\end{array}$ \\
\hline $\begin{array}{c}\text { Temperatura del fluido } \\
\left({ }^{\circ} \mathrm{C}\right)\end{array}$ & 50 & 90 \\
\hline $\begin{array}{c}\text { Viscosidad del petróleo, } \mu \\
\left(\mathrm{kg} \cdot \mathrm{h}^{-1} \cdot \mathrm{m}^{-1}\right)\end{array}$ & 72 & 20,16 \\
\hline $\begin{array}{c}\text { Densidad del petróleo, } \rho \\
\left(\mathrm{kg} \cdot \mathrm{m}^{-3}\right)\end{array}$ & 877 & 780 \\
\hline $\begin{array}{c}\text { Calor específico, } \mathrm{Cp} \\
\left(\mathrm{kcal} . \mathrm{kg}^{-1} \cdot{ }^{\circ} \mathrm{C}^{-1}\right)\end{array}$ & 0,5 & 0,5 \\
\hline $\begin{array}{c}\text { Conductividad térmica, } \mathrm{kf} \\
\left(\mathrm{kcal} \cdot \mathrm{m}^{-1} \mathrm{~h}^{-1} \cdot \mathrm{m}^{-2} .{ }^{\circ} \mathrm{C}^{-1}\right)\end{array}$ & 0,124 & 0,186 \\
\hline
\end{tabular}

Con vistas a establecer la magnitud en la cual el límite mínimo del coeficiente de transferencia de calor por convección, calculado por la Ecuación 5, debe ser disminuido debido a la disminución de presión dentro del tubo durante la reparación se emplea la ecuación de Bernoulli [25], presentada aquí como Ecuación 6. En esta ecuación fue ignorada la distribución no uniforme de la velocidad y la pérdida de energía de carga.

$$
z+p / \gamma+v^{2} / 2 g=c t e
$$

Donde: $\mathrm{z}$ - energía específica de posición; $\mathrm{p} / \tilde{\mathrm{a}}$ - energía

específica de presión del fluido en movimiento; $\mathrm{v}^{2} / 2 \mathrm{~g}$ energía cinética específica del líquido.
En el artículo fue considerado que el valor mínimo del coeficiente de transferencia de calor por convección es de 290 $\mathrm{W} \cdot \mathrm{m}^{-2} \cdot \mathrm{K}^{-1}$, el valor intermedio es de $365 \mathrm{~W} \cdot \mathrm{m}^{-2} \cdot \mathrm{K}^{-1}$ y el valor superior es de $440 \mathrm{~W} \cdot \mathrm{m}^{-2} \cdot \mathrm{K}^{-1}$, calculados a partir de las ecuaciones 5 y 6 . En la superficie externa del tubo (temperatura ambiente), el coeficiente asumido fue de $13 \mathrm{~W} \cdot \mathrm{m}^{-2} \cdot \mathrm{K}^{-1}$, de acuerdo con trabajos publicados por Cisilino [10].

\subsubsection{Organización de los datos para la solución de los modelos}

Con vistas a reducir el número de modelos a solucionar, las variables fueron organizadas empleando la matriz de un diseño de experimentos del tipo factorial, con tres variables y tres niveles en cada variable $\left(3^{3}\right)$. Esto arroja la necesidad de realizar 27 modelaciones o experimentos virtuales.

Las variables dependientes (variables respuestas) que se obtienen de la simulación con elementos finitos son: la máxima temperatura en la superficie interna del tubo $\left(\mathrm{T}_{\mathrm{MAX}}\right)$ y el tiempo de enfriamiento entre $800{ }^{\circ} \mathrm{C}$ y $500{ }^{\circ} \mathrm{C}$ (t8/5) de un punto localizado en la subzona de grano grueso de la zona afectada por el calor (ZAC).

Las Figuras 2 y 3 muestran los campos de temperatura sobre el modelo durante la deposición del cordón de filete que une el tubo y el refuerzo y la historia térmica de un punto situado en la subzona de grano grueso (situada aproximadamente por encima de $1200{ }^{\circ} \mathrm{C}$ y hasta la temperatura de sólido), de la zona afectada por el calor (ZAC).

La Tabla 4 muestra los resultados de las variables dependientes para cada una de las combinaciones entre las variables independientes.

Tabla 4. Resultados de la solución de los modelos de elementos finitos.

\begin{tabular}{|c|c|c|c|c|c|c|c|c|c|c|c|}
\hline \multirow[b]{2}{*}{$\begin{array}{c}\text { Estudio } \\
\mathbf{N}^{0}\end{array}$} & \multicolumn{3}{|c|}{$\begin{array}{c}\text { Variables } \\
\text { independientes }\end{array}$} & \multicolumn{2}{|c|}{$\begin{array}{c}\text { Variables } \\
\text { dependientes }\end{array}$} & \multirow[b]{2}{*}{$\begin{array}{c}\text { Estudio } \\
\mathbf{N}^{\circ}\end{array}$} & \multicolumn{3}{|c|}{$\begin{array}{c}\text { Variables } \\
\text { independientes }\end{array}$} & \multicolumn{2}{|c|}{$\begin{array}{c}\text { Variables } \\
\text { dependientes }\end{array}$} \\
\hline & $\mathbf{C E}$ & $\mathrm{CP}$ & TP & $\begin{array}{l}T_{\text {MAX }} \\
\left({ }^{\circ}{ }^{\circ}\right)\end{array}$ & $\begin{array}{c}\text { t } 8 / 5 \\
(s)\end{array}$ & & $\mathrm{CE}$ & $\mathbf{C P}$ & $\mathbf{T P}$ & $\begin{array}{l}T_{\text {MAX }} \\
\left({ }^{\circ} \mathrm{C}\right)\end{array}$ & $\begin{array}{c}\text { t } 8 / 5 \\
(s)\end{array}$ \\
\hline 1 & 1625 & 290 & 125 & 1273 & 8,3 & 15 & 810 & 440 & 200 & 565 & 2,38 \\
\hline 2 & 1625 & 440 & 200 & 1266 & 8,21 & 16 & 1625 & 440 & 125 & 1229 & 7,5 \\
\hline 3 & 1625 & 440 & 50 & 1150 & 5,45 & 17 & 810 & 290 & 125 & 551 & 1,96 \\
\hline 4 & 810 & 290 & 50 & 507 & 1,42 & 18 & 1625 & 290 & 50 & 1219 & 7,39 \\
\hline 5 & 810 & 365 & 125 & 532 & 1,5 & 19 & 1625 & 365 & 125 & 1241 & 7,47 \\
\hline 6 & 810 & 365 & 50 & 479 & 1,33 & 20 & 1217 & 365 & 50 & 840 & 3,73 \\
\hline 7 & 1217 & 440 & 200 & 971 & 6,28 & 21 & 810 & 440 & 125 & 516 & 1,45 \\
\hline 8 & 1217 & 365 & 125 & 913 & 5,08 & 22 & 1217 & 365 & 200 & 982 & 6,37 \\
\hline 9 & 810 & 440 & 50 & 457 & 1,26 & 23 & 810 & 290 & 200 & 627 & 3,65 \\
\hline 10 & 1625 & 365 & 200 & 1300 & 9,39 & 24 & 1625 & 365 & 50 & 1178 & 5,9 \\
\hline 11 & 1217 & 440 & 125 & 907 & 5,09 & 25 & 1625 & 290 & 200 & 1348 & 12,5 \\
\hline 12 & 1217 & 440 & 50 & 819 & 4,64 & 26 & 1217,5 & 290 & 50 & 863 & 3,93 \\
\hline 13 & 810 & 365 & 200 & 587 & 2,76 & 27 & 1217,5 & 290 & 200 & 1015 & 6,72 \\
\hline 14 & 1217 & 290 & 125 & 942 & 5,55 & & & & & & \\
\hline
\end{tabular}




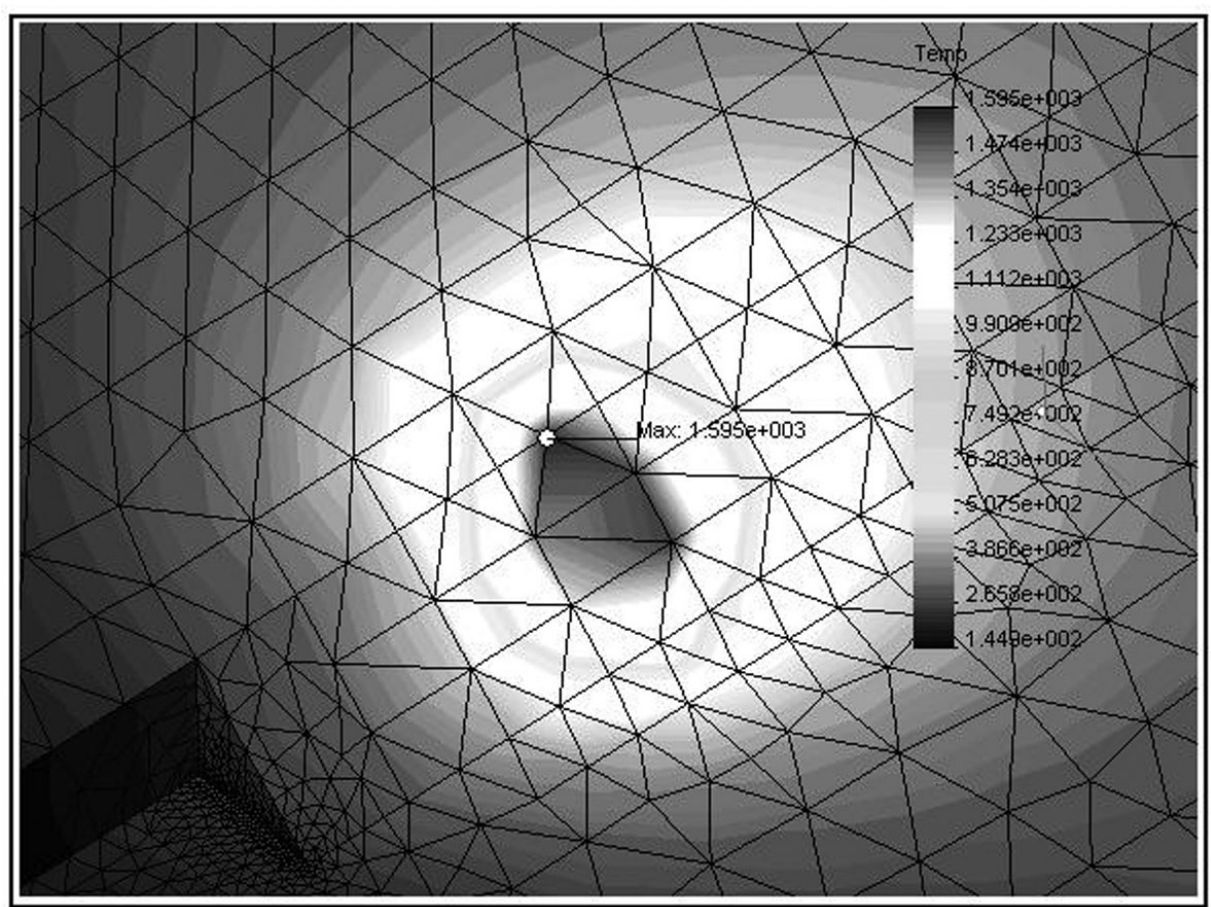

Figura 2. Campo de temperatura tras la solución del modelo de elementos finitos.

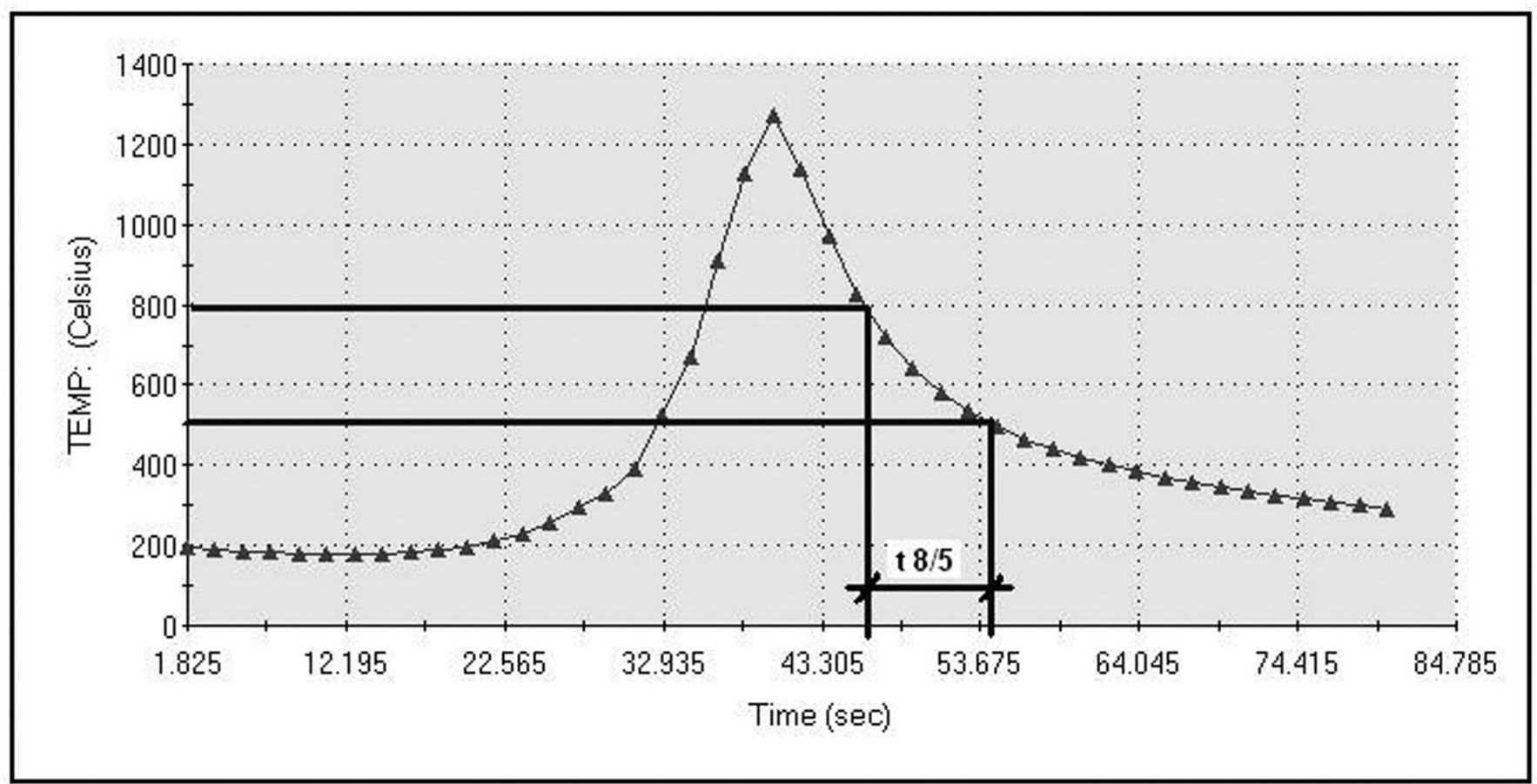

Figura 3. Determinación del t 8/5 a partir de los resultados del modelo de elementos finitos.

\section{Resultados y discusión}

Partiendo del comportamiento de las variables dependientes (máxima temperatura en la superficie interna del tubo y el tiempo de enfriamiento entre $800{ }^{\circ} \mathrm{C}$ y $500{ }^{\circ} \mathrm{C}$ ) puede predecirse la ocurrencia o no de perforaciones y agrietamiento en frío.

Para evitar las perforaciones, como fue mencionado antes, es necesario que $\mathrm{T}_{\text {MAX }}$ sea menor que $982{ }^{\circ} \mathrm{C}$ (también llamada temperatura de Batelle).

Empleando las Ecuaciones de la 7 a la 11, desarrolladas por Yurioka [26] especialmente para la estimación de la dureza máxima en aceros de tuberías, se puede calcular el t $8 / 5$ crítico. Para esto se iguala la dureza máxima obtenida en la ZAC $\left(\mathrm{HV}_{\mathrm{MAX}}\right)$, de la Ecuación 7, al valor límite de dureza (250 HV ó $350 \mathrm{HV}$ ) y se despeja el término que contiene al t8/5. 
$H V_{M A X}=442 C+99 C E_{I I}+206+\left(402 C-90 C E_{I I}+80\right) \arctan (x)$

$$
x=\frac{\log (t 8 / 5)-2,30 C E_{I}-1,35 C E_{I I}+0,882}{1,15 C E_{I}-0,673 C E_{I I I}-0,601}
$$

$$
C E_{I}=C+S i / 24+M n / 6+C u / 15+N i / 12+C r / 8+M o / 4+\Delta H
$$

$$
C E_{I I}=\mathrm{C}+\mathrm{Si} / 24+\mathrm{Mn} / 5+\mathrm{Cu} / 10+\mathrm{Ni} / 18+\mathrm{Cr} / 5+\mathrm{Mo} / 2,5+\mathrm{V} / 5+\mathrm{Nb} / 3
$$

$$
C E_{I I I}=\mathrm{C}+\mathrm{Mn} / 3,6+\mathrm{Cu} / 20+\mathrm{Ni} / 9+\mathrm{Cr} / 5+\mathrm{Mo} / 4
$$

Donde: $\mathrm{C}, \mathrm{Si}, \mathrm{Mn}, \mathrm{Cu}, \mathrm{Ni}, \mathrm{Cr}, \mathrm{Mo}, \mathrm{V}$ y Nb representan los porcientos en peso de los elementos químicos carbono, silicio, manganeso, cobre, níquel, cromo, molibdeno, vanadio y niobio; $\Delta \mathrm{H}$ representa un término introducido para tener en cuenta el fuerte efecto de endurecimiento que realiza el elemento químico boro, cuando está presente en el acero.

La dureza en la ZAC limitada a $350 \mathrm{HV}$ es una consideración muy común para evitar el agrietamiento en frío $[11,20,22]$. Cuando el hidrocarburo que es transportado se considera hidrocarburo amargo (hidrocarburo que contiene agua en fases líquida y/o vapor y ácido sulfhídrico), la dureza en la ZAC no puede exceder $250 \mathrm{HV}$ para evitar el agrietamiento en frío $[11,27]$.
Los resultados, mostrados en la Tabla 4, fueron procesados empleando STATGRAPHICS Centurión XV ver. 15.1.02 y SPSS ver. 11.5.1 y las Ecuaciones 12 y 13 fueron obtenidas:

$T_{M A X}=\exp (5,49827+0,0010388 \times C E-0,000418 \times C P+0,0010458 \times T P)$

Con una $\mathrm{R}^{2}$ ajustado de 0,969335 para un $95 \%$ de confianza.

$t^{8} / 5 \min =-3,58781+0,0069885 \times C E-0,00543707 \times C P+0,0158445 \times T P$

Con una $\mathrm{R}^{2}$ ajustado de 0,967676 para un $95 \%$ de confianza.

La idoneidad estadística de estas ecuaciones de regresión fue valorada a través del análisis de sus residuos. En este análisis se comprobó que los residuos de ambas ecuaciones cumplen las cuatro condiciones necesarias, que son: los residuos están distribuidos normalmente, tienen media cero y varianza constante, no están correlacionados con la variable dependiente y no están autocorrelacionados.

Durante el desarrollo del análisis de residuos fue rechazada una ecuación de regresión lineal, para relacionar a la temperatura máxima en la superficie del tubo $\left(\mathrm{T}_{\mathrm{MAX}}\right)$ y las variables dependientes, debido a falta de normalidad de los residuos y a correlación con la variable dependiente $\left(\mathrm{T}_{\mathrm{MAX}}\right)$, tal y como se muestra gráficamente en la Figura 4.
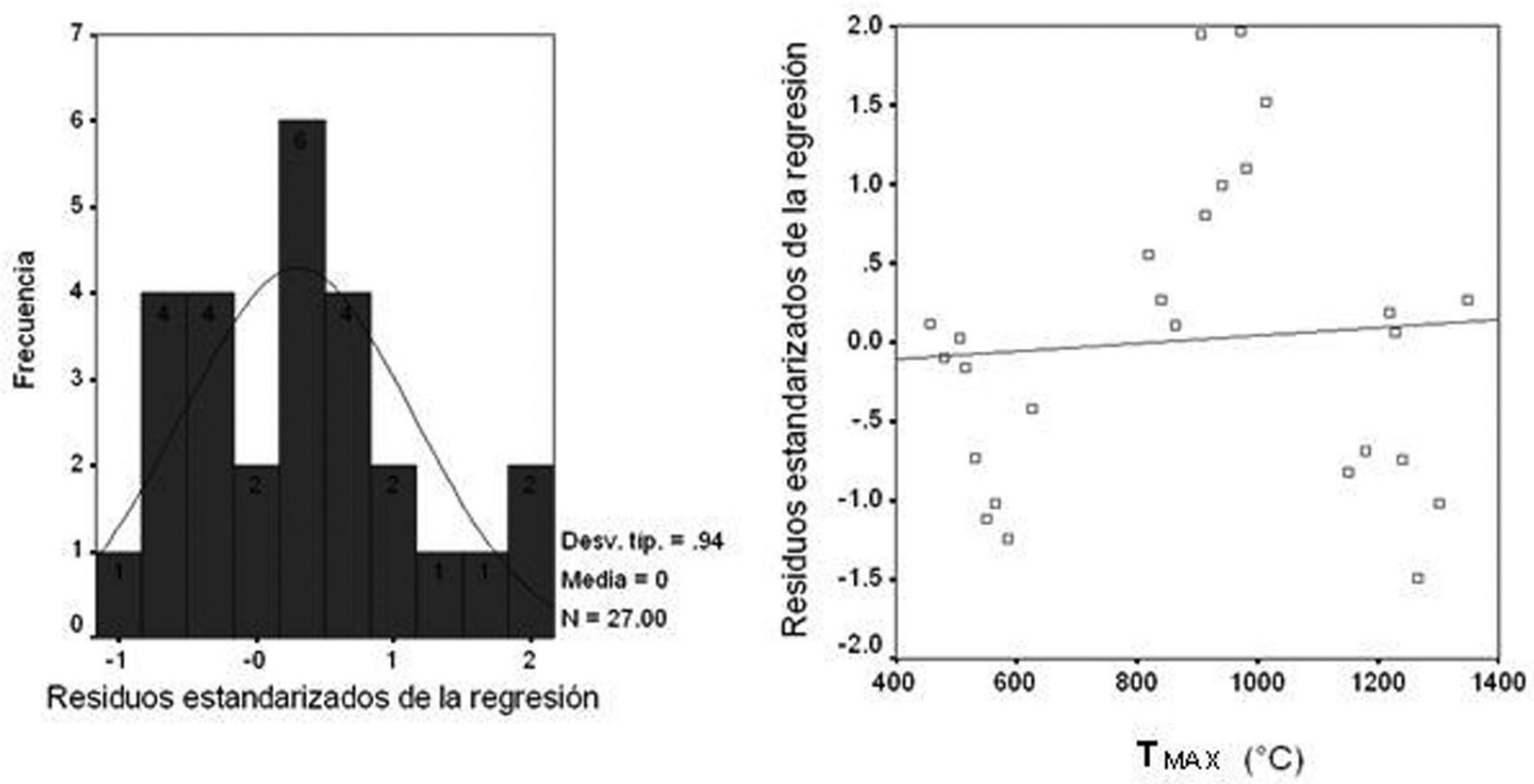

Figura 4. Resultados del análisis gráfico de los residuos, de la ecuación de regresión lineal rechazada para la $\mathrm{T}_{\mathrm{MAX}}$. 
Para un acero designado como X 65, empleado en tuberías, con una composición química como la mostrada en la Tabla 5 , se calcula el carbono equivalente según la fórmula del Instituto Internacional de Soldadura [28] y el t $8 / 5$ crítico o t8/5 necesario para obtener una dureza en la ZAC menor que 250 HV [26]; el cálculo de estos parámetros arrojó: $0.33 \%$ y 5,8 s respectivamente.

Tabla 5. Composición química de acero X 65 para tuberías [29].

\begin{tabular}{|c|c|c|c|c|c|c|c|c|c|}
\hline \% C & \% $\mathbf{~ S i}$ & \% Mn & \% Ni & \% Nb & \% V & \% Al & \% P & \% S & \% Cu \\
\hline 0,10 & 0,20 & 1,20 & 0,15 & 0,03 & 0,05 & 0,035 & 0,015 & 0.008 & 0,15 \\
\hline
\end{tabular}

Las condiciones que garantizan que en un tubo con la composición química anterior no ocurra, durante la soldadura en condiciones de servicio, perforaciones o agrietamiento en frío son establecidas en las Ecuaciones 14 y 15 :

$$
\begin{aligned}
& T_{M A X}<982^{\circ} \mathrm{C} \\
& t^{8} / 5>6 s
\end{aligned}
$$

$\mathrm{Si}$ se desarrollan las condiciones mostradas en las Ecuaciones 14 y 15, a partir de las Ecuaciones 12 y 13, se obtienen las Ecuaciones 16 y 17 :

$\exp (5,49827+0,001038 \times C E-0,000418 \times C P+0,0010458+T P)<982^{\circ} \mathrm{C}$

\section{$3,5878+0,0069885 C E-0,00543707 C P+0,0158445 T P>6 s$}

En ambas ecuaciones (16 y 17) se despeja la variable calor de entrada (CE), quedando entonces que el $\mathrm{CE}$ es una función del coeficiente de película (CP) y de la temperatura de precalentamiento (TP), lo que podemos expresar como: $\mathrm{CE}=\mathrm{f}$ (CP, TP).

Si estas ecuaciones (16 y 17) se evalúan para valores del coeficiente de transferencia de calor por convección (CP) entre $290 \mathrm{~W} \cdot \mathrm{m}^{-2} \cdot \mathrm{K}^{-1}$ y $440 \mathrm{~W} \cdot \mathrm{m}^{-2} \cdot \mathrm{K}^{-1}$ y solamente para una temperatura de precalentamiento de $200{ }^{\circ} \mathrm{C}$, se obtiene un gráfico como el mostrado en la Figura 5.

En este gráfico el área debajo de la recta que representa las soluciones de la Ecuación 16, se corresponde a una zona de no ocurrencia de perforaciones en la pared del tubo. Por otra parte, el área encima de la recta que representa las soluciones de la Ecuación 17, se corresponde a la zona de no ocurrencia de agrietamiento en frío.

$\mathrm{Al}$ considerarse conjuntamente el papel de ambas rectas, aparecen 3 zonas que describen comportamientos diferentes al ejecutarse la soldadura:

Zona I: Los pares de valores de CE y CP que queden contenidos dentro de esta zona, no representan soluciones de la Ecuación 16 y sí son soluciones de la Ecuación 17. Hay riesgo de que se perfore la pared del tubo. Esta perforación ocurre en el momento que se desarrolla la reparación y puede ir acompañada de un incendio o una explosión.
Zona II: Los pares de valores de CE y CP que queden contenidos en esta zona (área sombreada), representan soluciones de las dos ecuaciones analizadas (16 y 17). No ocurre la perforación de la pared del tubo y tampoco agrietamiento en frío. Esta zona es la que se puede considerar como preferida para hacer la reparación, en condiciones de servicio, de una tubería.

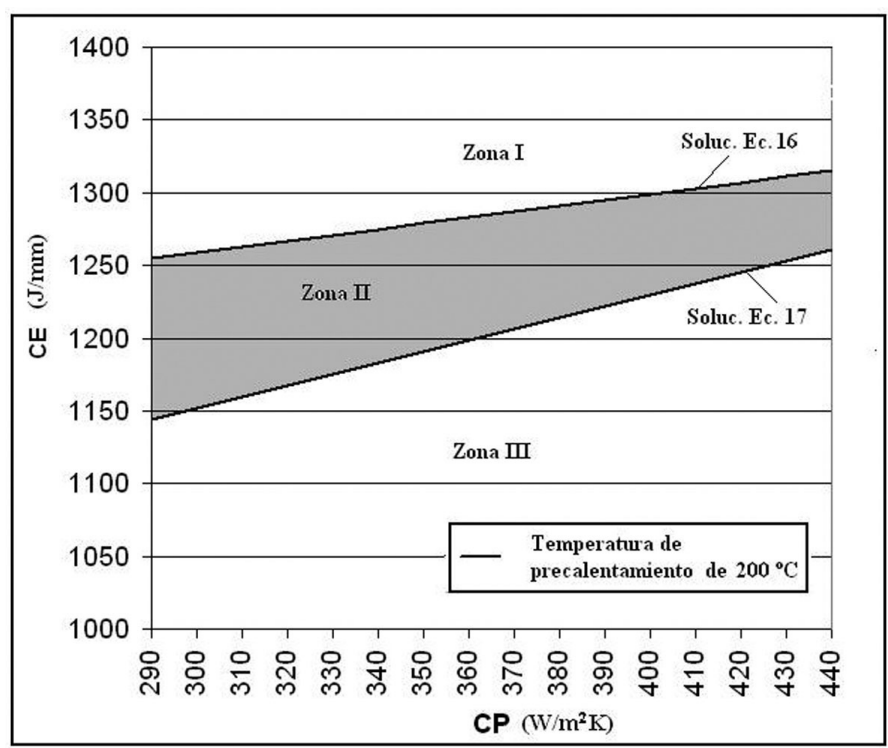

Figura 5. Zonas del nomograma para la determinación del aporte térmico que garantice que el $t 8 / 5>6$ s y que la

$$
\mathrm{T}_{\text {MAX }}<982^{\circ} \mathrm{C} \text {. }
$$

Zona III: Los pares de valores de CE y CP contenidos en esta zona, representan soluciones de la Ecuación 16, pero no de la Ecuación 17. Hay riesgo de ocurrencia de agrietamiento en frío. Este fenómeno no se manifiesta inmediatamente, pueden pasar días, meses e incluso años antes de que la grieta crezca lo suficiente para que la tubería falle catastróficamente. Si el sistema de inspección de la tubería funciona adecuadamente, posiblemente se detecte la grieta antes de llegar a la magnitud crítica y no ocurra la falla catastrófica.

Si las funciones de CE, obtenidas a partir de las Ecuaciones 16 y 17 , son evaluadas para los diferentes valores de CP (entre $290 \mathrm{~W} \cdot \mathrm{m}^{-2} \cdot \mathrm{K}^{-1}$ y $\left.440 \mathrm{~W} \cdot \mathrm{m}^{-2} \cdot \mathrm{K}^{-1}\right)$ y para los valores de TP $\left(50{ }^{\circ} \mathrm{C}\right.$, $100{ }^{\circ} \mathrm{C}, 150{ }^{\circ} \mathrm{C}$ y $200{ }^{\circ} \mathrm{C}$ ) se obtiene un gráfico (o nomograma) como el presentado en la Figura 6. 


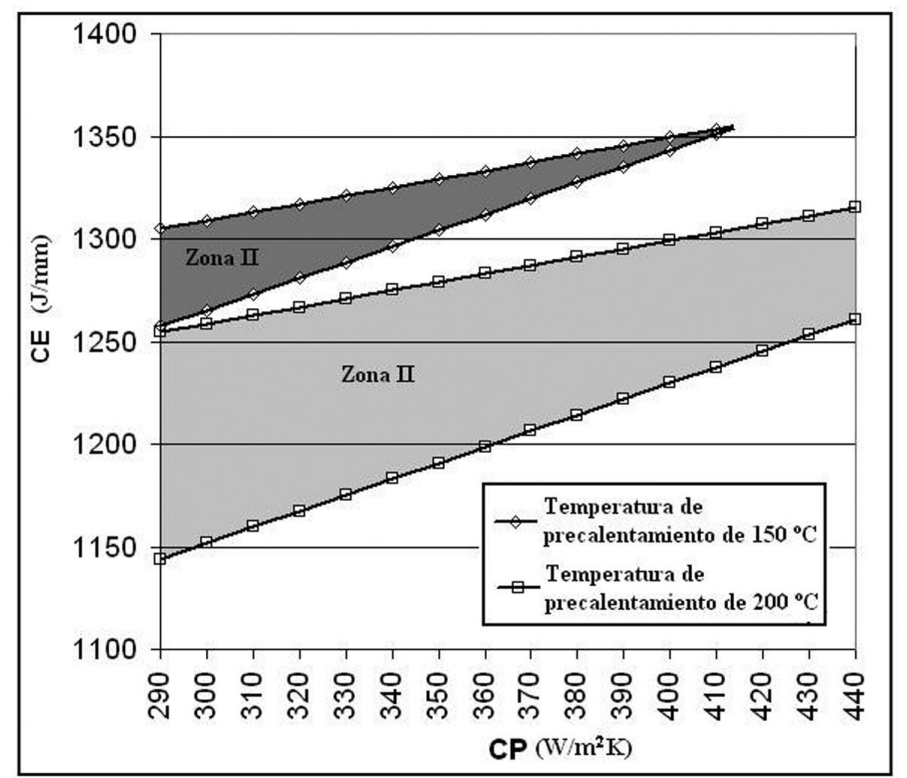

Figura 6. Nomograma para la determinación del aporte térmico, para diferentes valores de TP, para garantizar un t8/5>6s y

$$
\mathrm{T}_{\text {MAX }}<982^{\circ} \mathrm{C} \text {. }
$$

En este se puede apreciar que para temperaturas de precalentamiento (TP) de $50{ }^{\circ} \mathrm{C}$ y $100{ }^{\circ} \mathrm{C}$ no hay soluciones comunes a ambas ecuaciones. También se aprecia que el incremento de la temperatura de precalentamiento puede ampliar la zona II del diagrama, permitiendo que tuberías con condiciones de disipación del calor más intensas (mayor coeficiente de transferencia de calor por convección, CP) puedan ser soldadas sin riesgo; por supuesto la soldadura con mayores valores de temperatura de precalentamiento (TP) obliga a un menor valor de calor de entrada (CE) de la soldadura, para que no ocurra la perforación de la pared del tubo.

Para un valor definido del coeficiente de transferencia de calor por convección (CP) que, como se apreció en la Ecuación 5 , es una función de las propiedades del fluido, de las condiciones de flujo y de las dimensiones de la tubería; la elección de la magnitud del calor de entrada (CE) y de la temperatura de precalentamiento (TP) a emplear en el proceso de soldadura, debe tomar en cuenta las siguientes recomendaciones:

1. Para tuberías relativamente nuevas y que la causa de su daño haya sido la acción humana durante la transportación, montaje u explotación del tubo; puede elegirse una combinación tal que la temperatura de precalentamiento sea la menor posible (tecnológicamente es más sencillo soldar sin precalentamiento o con un valor bajo de esta temperatura), ya que en este caso la capacidad de atrapamiento de hidrógeno por parte del material del tubo es baja y combinado con el uso de electrodos de bajo hidrógeno, no es fundamental la liberación de hidrógeno desde el metal del tubo. El calor de entrada puede seleccionarse también en el límite inferior del rango posible.

2. Para tuberías viejas, en las que la causa de daño esté relacionada al agrietamiento inducido por hidrógeno, a la corrosión bajo tensión o a la corrosión, entre otros mecanismos; puede elegirse una combinación de valores de calor de entrada y temperatura de precalentamiento tal que se aporte la mayor cantidad de energía calorífica posible con vistas a eliminar la mayor cantidad posible del hidrógeno residual contenido en la estructura del metal (este hidrógeno penetró en el metal durante la vida de servicio y también durante la soldadura, a pesar de emplearse electrodos de bajo hidrógeno, debido a la gran capacidad de atrapamiento de hidrógeno que tiene el metal del tubo tras larga un largo período de explotación).

En el caso en que el t 8/5 crítico del material sea superior a 7 segundos (valor a sustituir en la Ecuación 17, en vez de 6 segundos), es imposible obtener soluciones comunes a ambas ecuaciones (Ecuaciones 16 y 17); por lo que no es posible garantizar, simultáneamente, la no ocurrencia de perforaciones y de agrietamiento en frío. En estos casos el fenómeno crítico es la perforación de la pared del tubo y la selección del calor de entrada se hace en base a este criterio. La Figura 7 muestra un nomograma que define la ocurrencia o no de perforaciones, al soldar el tubo sin precalentamiento.

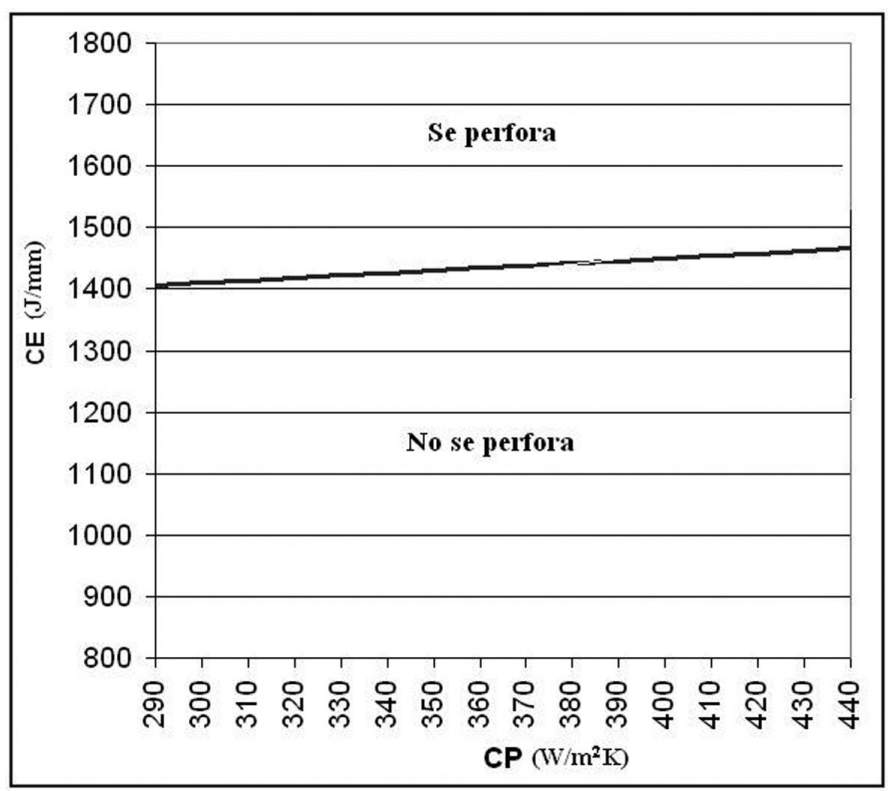

Figura 7. Nomograma para la determinación del aporte térmico para garantizar una $\mathrm{T}_{\mathrm{MAX}}<982^{\circ} \mathrm{C}$, sin emplear precalentamiento del tubo.

Independientemente de que predomine el criterio de evitar la perforación del tubo (en aquellos casos en que no se tengan soluciones comunes a las ecuaciones analizadas), no se descuida la posible ocurrencia de agrietamiento en frío y esta sensibilidad es disminuida extremando las medidas de limpieza de las superficies a soldar; mediante la calcinación de los electrodos básicos a emplear en la soldadura; disminuyendo al máximo posible las tensiones residuales del proceso de soldadura (adecuada secuencia de soldadura, tratamiento de alivio de tensiones). También deben estrecharse los períodos entre inspecciones, con vistas a detectar posibles grietas en frío, en longitudes por debajo de la longitud crítica.

Los resultados obtenidos en la presente investigación, 
acerca del límite en la magnitud del calor de entrada (CE) para que no ocurra la perforación del tubo, son comparables a resultados obtenidos en trabajos de investigación por el Institute of Materials Engineerings of Australia [30]. Estos trabajos expresan que, para espesores de tubo de hasta $6 \mathrm{~mm}$, el calor de entrada máximo es del orden de $1,50 \mathrm{~kJ} \cdot \mathrm{mm}^{-1}$ a $1,60 \mathrm{~kJ} \cdot \mathrm{mm}^{-1}$ aproximadamente, desarrollando la soldadura a la temperatura del fluido (sin precalentar). Esta comparación indica un ligero carácter conservador de los resultados obtenidos en la presente investigación, pero ante las severas consecuencias que puede traer una perforación durante la ejecución de la soldadura, este carácter no demerita el valor de las ecuaciones y nomogramas obtenidos.

\section{Conclusiones}

Los resultados obtenidos en esta investigación, permitieron establecer ecuaciones de regresión que relacionan al calor de entrada de la soldadura, la temperatura de precalentamiento y el coeficiente para la transferencia de calor por convección, con la temperatura máxima en la superficie interna del tubo y el tiempo de enfriamiento entre $800^{\circ} \mathrm{C}$ y $500{ }^{\circ} \mathrm{C}$ de un punto situado en la ZAC. Estas ecuaciones poseen soluciones comunes para aceros con un t $8 / 5$ crítico menor que 7 segundos y tal que se garantice que la temperatura máxima en la superficie interna del tubo no exceda $982{ }^{\circ} \mathrm{C}$.

A partir de la solución de las ecuaciones obtenidas, es posible seleccionar niveles de energía calorífica a aportar durante el desarrollo del proceso de soldadura, tal que se evite, simultáneamente, la ocurrencia de perforaciones en la pared del tubo y de agrietamiento en frío en la ZAC.

Los nomogramas creados a partir de las ecuaciones generales obtenidas presentan gran valor práctico para la selección de las condiciones en que se ha de desarrollar el proceso de soldadura. Las ecuaciones y nomogramas son válidos para los siguientes intervalos de valor de las variables consideradas:

1. Temperatura de precalentamiento entre $50{ }^{\circ} \mathrm{C}$ y $200{ }^{\circ} \mathrm{C}$.

2. Coeficiente de transferencia de calor por convección entre $290 \mathrm{~W} \cdot \mathrm{m}^{-2} \cdot \mathrm{K}^{-1}$ y $440 \mathrm{~W} \cdot \mathrm{m}^{-2} \cdot \mathrm{K}^{-1}$.

3. Calor de entrada de la soldadura entre $810 \mathrm{~J} \mathrm{~mm}^{-1}$ y 1625 $\mathrm{J} . \mathrm{mm}^{-1}$.

4. Aceros de baja aleación de acuerdo a la especificación API $5 \mathrm{~L}$.

5. Espesor del tubo de $6.4 \mathrm{~mm}$.

\section{Referencias bibliográficas}

[1] PAPADAKIS, G. Major Hazard pipelines, a comparative study of onshore transmission accidents. Journal of Loss Prevention in the Process Industries, v.12, p. 91-107, 1999.

[2] KIEFNER, J.F., TRENCH, Ch. J. Oil Pipelines Characteristics and Risk Factors: Illustrations from the Decade of Constructions. Report for American Petroleum Institute, USA, 2001.

[3] PALMER - JONES, R.; PAISLEY, D. Repairing Internal Corrosion Defects in Pipelines - A case study. In: INTERNATIONAL PIPELINES REHABILITATION AND
MAINTENANCE CONFERENCE, $4^{\circ}, 2000$, Prague.

[4] FAZZINI, P. G; OTEGUI, J. L. Influence of old rectangular repair patches on the burst pressure of a gas pipeline. International Journal of Pressure Vessels and Piping, v.83, p. 27-34, 2006.

[5] AMERICAN PETROLEUM INSTITUTE. API RP 570: Inspection, repair, alteration and rerating of in - service piping system, Second Edition - Addendum 2, USA, 2001. 111 p.

[6] EMPRESA CUBANA DEL PETROLEO. Regulación para el transporte de hidrocarburos gaseosos por ducto, Código DO -DU/R 0101, Revisión 00, Cuba, 2000. 81 p.

[7] BAEK, J., KIM, W., KIM, Y. Comparative study for various repair methods of in-service pipeline using full scale burst test. In: WORLD GAS CONFERENCE, 23ㅇ 2006, Amsterdam, Netherlands. $11 \mathrm{p}$.

[8] BRUCE, W. A., HARWIG, D. D., HARRIS, I. D., GORDON, J. R., SULLIVAN, M and NEARY, C. Development of internal repair technology for gas transmission pipelines. GTI CONFERENCE AND EXHIBITION, 2004, Phoenix, Arizona, USA.

[9] AMERICAN PETROLEUM INSTITUTE. API Standard 1104: Welding of Pipelines and Related facilities, Nineteenth Edition, USA, 1999, $82 \mathrm{p}$.

[10] CisilinO, A. P.; CHAPETTI, M. D.; OTEGUI, J. L. Minimum thickness for circumferential sleeve repair fillet welds in corroded gas pipelines. International Journal of Pressure Vessel and Piping, v. 79, p. $67-76,2002$.

[11] BANG, I. W., SON, Y. P., OH, K. H., KIM, Y. P., KIM, W. S. Numerical Simulation of Sleeve Repair Welding of InService Gas Pipelines. Welding Research Supplement, Welding Journal, p. 273 - 282, December 2002.

[12] ODDY, A. S., McDILL, J. M. J. Burn through prediction in pipeline welding. International Journal of Fracture, v. 97, n. 4, p. $249-261,1999$.

[13] YURIOKA, N. NIPPON STEEL Weldability Calculation Master Code, updated on April, 2007, Version 1.5.

[14] PAVLOV, K. F.; ROMANKOV, P. G.; NOSKOV, A. A. Problemas y ejemplos para el curso de operaciones básicas y aparatos en tecnología química, Editorial MIR, Moscú, 1982.

[15] RAMOS, F., DUFFUS, A. RODRÍGUEZ, M., DÍAZ, E. M., POZO, J. A. Modelo de elementos finitos para determinar el campo de temperatura en una costura de filete. Ingeniería: Revista Académica de la Facultad de Ingeniería, de la Universidad Autónoma de Yucatán, v. 11, n. 3, p. 33 - 42, 2007.

[16] POORHAYDARI, K., PATCHETT, B. M., IVEY, D. G. Estimation of cooling rate in the welding of plates with intermediate thickness. Welding Research Supplement, Welding Journal, p. 149 - 155, October 2005.

[17] AMERICAN WELDING SOCIETY. ANSI / AWS A 5.5: Specification for Low Alloy Steel Covered Welding Electrodes, USA, 1996, $55 \mathrm{p}$.

[18] AMERICAN WELDING SOCIETY. Welding Handbook: Welding Science and Technology, Ninth edition, Volume 1, USA, 2001.

[19] AMERICAN WELDING SOCIETY. ANSI / AWS D1.1. Structural Welding Code - Steel, USA, 2000.

[20] KOU, S. Welding Metallurgy, Second edition, Wiley Interscience, USA, 2003. 428 p. 
[21] KURZYDLOWSKI, K. J., NYKYFORCHYN, H., LUNARSKA, E., TSYRULNYK, O. and CIUPINSKI, Ł. Susceptibility to stress corrosion in transport oil pipeline steels exposed to long time service conditions. 2001. Extracted on June 17 of 2004 from web site http://www.hallf.kth.se/forskning/ ecf15/ ECF-proceedings/Kurzydlowski\%20K-J\%20etal.pdf.

[22] QUESADA, H. J., ZALAZAR, M.; ASTA, E. P.. Ensayos de fisuración en frío aplicados en aceros de alta resistencia. JORNADAS SAM - CONAMET - AAS, septiembre 2001, Argentina.

[23] YURIOKA, N. Physical Metallurgy of Steel Weldability. ISIJ International, v. 6, n. 41, p. $566-570.2001$.

[24] FAIRES, V. M. Termodinámica, Edición Revolucionaria, La Habana, Cuba, 1975. 807 p.

[25] NEKRASOV, B. Hidráulica, Edición Revolucionaria, La Habana, Cuba, 1976.

[26] NOLAN. D; STERJOVSKI, Z., DUNNE. D. Hardness prediction models based on HAZ simulation for in - service welded pipeline steels. Science and Technology of Welding and Joining, v. 6, n. 10, p. $681-694.2005$.

[27] PETRÓLEOS MEXICANOS. NRF-001-PEMEX-2000: Tubería de acero para recolección y transporte de Hidrocarburos amargos, México, 2000.

[28] INSTITUTO INTERNACIONAL DE SOLDADURA. Nota sobre el carbono equivalente. Revista de Soldadura, v. 20, n. 3, 1990.

[29] MITTAL STEEL SOUTH AFRICA. Composiciones químicas de los aceros de tuberías. Extraido del sitio web: www.arcelormittal.com

[30] GRACE, P. In - service welding of steel pipelines. SECOND NATIONAL STEEL CONFERENCE, 2003, Australia. 